\author{
Marquette University \\ e-Publications@Marquette
}

$12-14-2017$

\title{
Load Balancing with Energy Storage Systems Based on Co- Simulation of Multiple Smart Buildings and Distribution Networks
}

\author{
Shaun Duerr \\ Marquette University \\ Cristinel Ababei \\ Marquette University, cristinel.ababei@marquette.edu \\ Dan M. Ionel \\ University of Kentucky
}

Follow this and additional works at: https://epublications.marquette.edu/electric_fac

Part of the Computer Engineering Commons, and the Electrical and Computer Engineering Commons

\section{Recommended Citation}

Duerr, Shaun; Ababei, Cristinel; and Ionel, Dan M., "Load Balancing with Energy Storage Systems Based on Co-Simulation of Multiple Smart Buildings and Distribution Networks" (2017). Electrical and Computer Engineering Faculty Research and Publications. 318.

https://epublications.marquette.edu/electric_fac/318 
Marquette University

e-Publications@Marquette

\section{Electrical and Computer Engineering Faculty Research and Publications/College of Engineering}

This paper is NOT THE PUBLISHED VERSION; but the author's final, peer-reviewed manuscript. The published version may be accessed by following the link in the citation below.

2017 IEEE 6th International Conference on Renewable Energy Research, (November, 2017). DOI. This article is (C) Institute of Electrical and Electronic Engineers (IEEE) and permission has been granted for this version to appear in e-Publications@Marquette. IEEE does not grant permission for this article to be further copied/distributed or hosted elsewhere without the express permission from IEEE.

\section{Load Balancing with Energy Storage Systems Based on Co- simulation of Multiple Smart Buildings and Distribution Networks}

\section{Shaun Duerr}

Electrical and Computer Engr., Marquette University, Milwaukee WI

Cristinel Ababei

Electrical and Computer Engr., Marquette University, Milwaukee WI

Dan M. Ionel

Electrical and Computer Engr., University of Kentucky, Lexington KY

\section{IEEE Keywords}

Buildings, Load management, Tools, Energy storage, Optimization, Load modeling, Energy consumption 


\section{Abstract:}

In this paper, we present a co-simulation framework that combines two main simulation tools, one that provides detailed multiple building energy simulation ability with Energy-Plus being the core engine, and the other one that is a distribution level simulator, Matpower. Such a framework can be used to develop and study district level optimization techniques that exploit the interaction between a smart electric grid and buildings as well as the interaction between buildings themselves to achieve energy and cost savings and better energy management beyond what one can achieve through techniques applied at the building level only. We propose a heuristic algorithm to do load balancing in distribution networks affected by service restoration activities. Balancing is achieved through the use of utility directed usage of battery energy storage systems (BESS). This is achieved through demand response (DR) type signals that the utility communicates to individual buildings. We report simulation results on two test cases constructed with a 9-bus distribution network and a 57-bus distribution network, respectively. We apply the proposed balancing heuristic and show how energy storage systems can be used for temporary relief of impacted networks.

\section{SECTION I.}

\section{Introduction}

The building sector is the largest energy consumer in the world. For instance, buildings in the US account for more than 40 percent of the total energy consumption and greenhouse gas emissions ${ }^{[1]}$. There are two main approaches to address the problem of increasingly large energy consumption in buildings. On one hand, old buildings could be retrofitted by upgrading and replacing energyconsuming equipment in order to increase energy efficiency. However, this could be very costly despite the potential long term benefits. According to a study conducted by the Rockefeller Foundation, complete retrofit of pre-1980 buildings in the US would require $\$ 279$ billion ${ }^{[2]}$.

On the other hand, one can seek novel methods to reduce energy consumption in buildings through better operation and energy-aware changes in users' consumption behavior. Such methods are appealing especially because they do not require huge up-front capital investments. However, this is a challenging problem whose complexity is further exacerbated in the context of demand response (DR). Significant prior work has been done on the problem of DR in order to reduce, shed or shift electricity use to improve electric grid reliability and to manage costs. Integration of electric batteries or other energy storage systems (ESS), especially of the thermal type, within these DR studies represents a future trend that has been less studied. Furthermore, the deployment of renewable and distributed energy generation, especially photovoltaics (PV), is growing rapidly and is expected to make a substantial impact ${ }^{[3],[4]}$. Decentralized energy systems using renewable energy sources are increasingly considered with the use of energy storage ${ }^{[5]}$. Also, we find previous studies that explored the incorporation of more energy storage through placement of energy storage using techniques based on multidimensional scaling and clustering [6] and showed the economic advantages of hybrid systems ${ }^{[7]}$.

The work presented in this paper is an effort to answer the question of whether the interaction between the distribution network and buildings can be exploited to achieve better operation and 
optimizations beyond what one can achieve through techniques applied at the single building level only. To do that, we develop a co-simulation framework that combines two main simulation tools, one that provides detailed multiple building energy simulation ability with EnergyPlus being the core engine, and the other one that is a distribution level simulator, Matpower. Our hypothesis is that additional energy and cost savings can be achieved if we looked at multiple buildings at the same time and handle them within a single model rather than applying locally, at the building level, existing optimization techniques. The proposed co-simulation framework can provide the utility operators a tool capable of looking deeper into the interaction between buildings and distribution networks within a smart grid context, and which could be used to achieve further energy and cost savings through better operation.

\section{SECTION II.}

\section{Related Work}

The majority of previous work on building energy simulation focus on individual buildings without the ability to han- dle multiple buildings within the same simulation. However, the importance of incorporating in the simulation multiple interconnected building systems together with the electrical distribution grid has been recently demonstrated by European groups for net-zero buildings with PV generators ${ }^{[11]}$. They developed Integrated District Energy Assessment by Simulation (IDEAS), a Modelica library for the integrated modeling and simulation of buildings and districts. IDEAS can describe the built environment, energy consumption and supply, and networks and control in just one model, giving rise to a more effective analysis and better control of the energy system under consideration ${ }^{[11]}$. Other researchers also recognize the importance of the impact of building energy consumption control actions on the grid, because these actions can lead to supply/demand imbalance and voltage/frequency deviation and thus, threaten grid stability ${ }^{[12]}$. Thus, they illustrate a method for measuring the effects of individual building management tools on the grid and provide this information to the individual building simulation tools and, where warranted, to the building operators to help find corrective actions. Similarly, the authors of ${ }^{[13]}$ recognize that wind turbines and energy storage systems can be shared by the whole microgrid community to reduce the investment cost for each user. They propose a modeling framework, which coordinates demand response (DR) and distributed energy resource (DER) management with optimization, in order to reduce the overall energy consumption cost in the community.

Recently, the study in ${ }^{[14]}$ introduced, SmartBuilds, a district level multiple buildings energy simulation framework. The tool was used to simulate multiple buildings (up to 16 buildings) concurrently in order to asses and seek operating schedules for battery energy storage systems (BESS) that minimize the overall energy consumption at the district level. The authors of ${ }^{[15]}$ proposed an agent-based approach to optimize the inter-operation of smart grid building energy management systems (BEMS). Through integrated simulations they showed that the voltage profile of the feeder can be improved, while ensuring acceptable comfort levels in buildings. The study in ${ }^{[16]}$ extends capability of EnergyPlus to cosimulate a campus with multiple buildings connected to a chilled water distribution to a central chiller plant with control systems in MATLAB. They utilize the virtual campus test-bed to evaluate the performance of several demand response strategies and describe a coordinated demand response 
scheme which can lead to load curtailment during a demand response event while minimizing thermal discomfort. Finally, the authors of ${ }^{[17]}$ introduced the under-development openBuildNet simulation tool, which is a co-simulation platform that provides a framework for large-scale distributed control and simulation of complex multi-agent systems. They reported that the openBuildNet was demonstrated in a large-scale distributed simulation of a distribution grid of 47 buses.

Our work is similar to these recent studies in that we also focus on multiple buildings at the same time. However, we are proposing a co-simulation approach involving multiple buildings energy simulations and distribution network simu- lations to provide a framework for district level optimizations performed on the utility side. Such optimizations can include distribution network reconfiguration for load balancing, loss reduction, service restoration, etc. To the best of our knowledge, this has not been investigated so far.

\section{SECTION III.}

\section{Co-Simulation Framework for Multiple Buildings Energy and Distribution Network}

\section{Simulations}

\section{A. Proposed Co-Simulation Framework}

The block diagram of the proposed co-simulation framework coded in MATLAB is shown in Fig. 1. The multiple buildings energy simulation component is capable of launching multiple concurrent EnergyPlus simulation instances, one per each of the buildings $\left(b_{1}, b_{2}, \ldots, b_{n}\right)$, which are part of the test case. These buildings represent loads attached to the buses of the distribution network. EnergyPlus is one of the most popular and widely used building energy modeling tool developed by DOE and Lawrence Berkeley National Labs ${ }^{[9]}$. It models heating, cooling, lighting, ventilation, other energy flows, and water use. It was developed to provide an integrated simulation for accurate temperature and comfort prediction. 


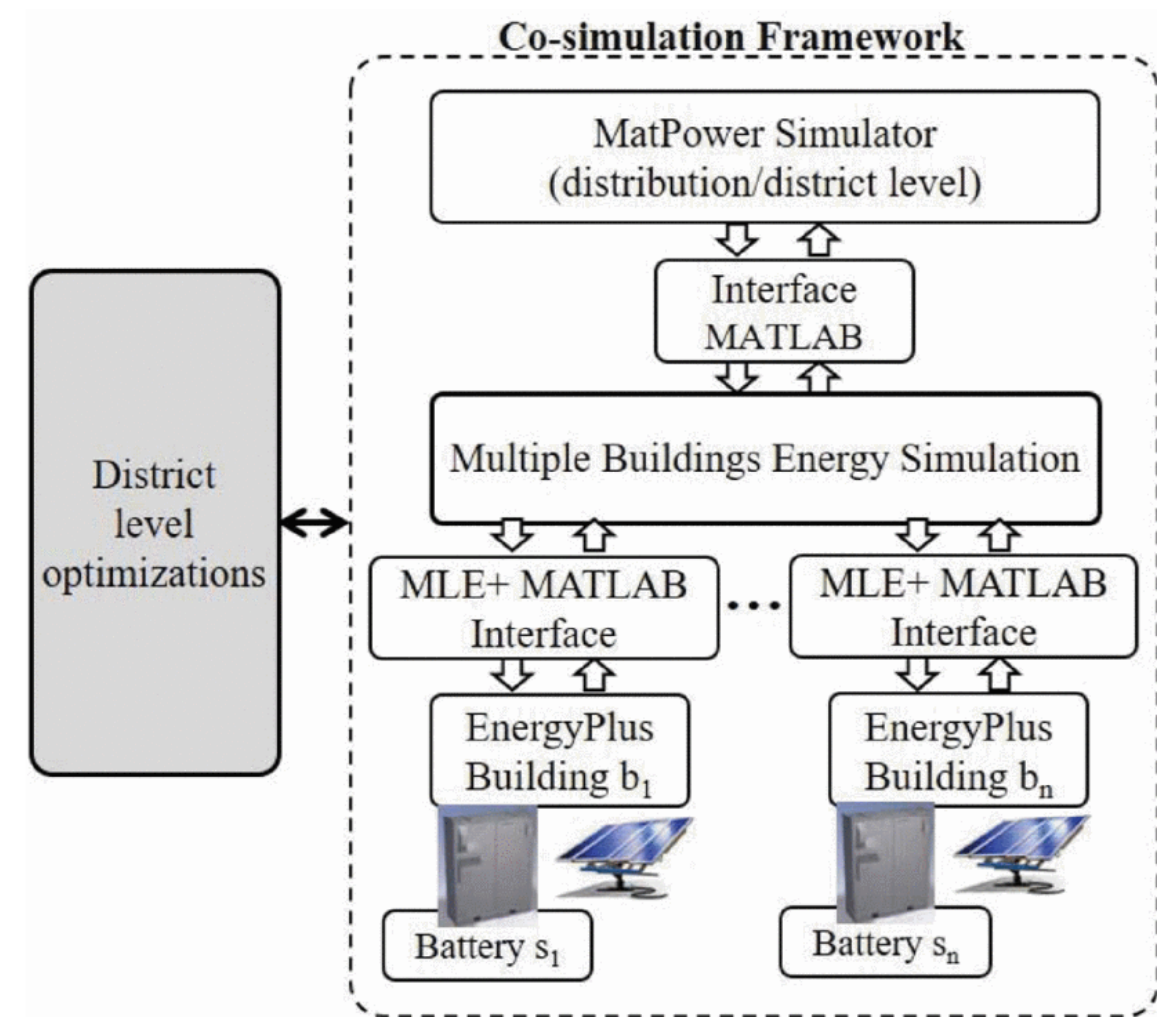

Fig. 1. Block diagram of the proposed co-simulation framework.

The capability of launching multiple such instances is facilitated through the use of an interface provided by the MLE+ building level optimization tool ${ }^{[18]}$, and executed using MATLAB's parallel processing toolbox. This tool provides the mechanics to launch and communicate with EnergyPlus simulator from within MATLAB. Each of the simulated buildings can include a battery as the energy storage system as well as a PV array as the renewable energy source. The EnergyPlus simulations can consider scheduling of energy consumption from batteries. Each of the EnergyPlus simulations can be paused in order to gather snapshot information about the status of the corresponding building and/or to input new controls, for example, according to the user provided battery consumption schedule for energy peak shaving purposes. This can be done at intervals of a few minutes, up to 60 minutes, for a total simulation time ranging between $24 \mathrm{~h}$ and one year. However, in this paper, we conduct simulations for $24 \mathrm{~h}$ only and a control period or time step of 5 minutes.

Depending on how buildings switch on or off their consumption from battery energy storage of other renewable resources, the impact on the feeder can vary widely and change rapidly. The changing load exercises the distribution network differently, and may shift its operation into modes that are not necessarily optimal, for example from load balancing or minimal losses perspectives. Therefore, at the distribution network level, we employ the popular Matpower ${ }^{[10]}$ simulation tool. Matpower is a tool for solving power flow and optimal power flow problems.

Matpower can be launched multiple times throughout the $24 \mathrm{~h}$ simulated time. So, the Matpower tool operates at a different timescale than the EnergyPlus tool, which is executed only once per building per 
$24 \mathrm{~h}$ simulated time. Because Matpower is executed multiple times during the $24 \mathrm{~h}$, it can effectively monitor the status of the distribution network. When significant changes occur in the load of the distribution network, various optimization techniques can be called in, which in turn can dictate desirable changes to the network. Such changes can include closing tie switches or opening sectionalizing switches, or they can represent other types of DR signals sent to individual consumers. Examples of optimization techniques include load balancing and network reconfiguration with objectives such as loss reduction. The block component on the left hand side in Fig. 1 encapsulates these optimization techniques, which must be implemented and integrated with the co-simulation framework.

The usefulness of the proposed co-simulation framework lies especially in the ability to perform investigation into such optimization techniques, quantify their impact, refine them, or seek new techniques that can help save energy, reduce costs, improve the distribution network reliability, introduce increasing amounts of renewable sources with minimal impact, and generally help to better operate the distribution network as a whole. An example of such optimization techniques is discussed in the next section.

\section{B. Load Balancing at Distribution Network Level}

In this section, we describe a load balancing technique that we have implemented and integrated in the co-simulation framework. We use this technique to illustrate the usefulness of the proposed cosimulation framework as a platform to evaluate distribution network operation modes before they are actually deployed in practice, as the strength of the presented co-simulation framework lies in its ability to simulate a functioning smart grid. Indeed, within the framework, we are able to co-simulate a utility, multiple smart buildings, energy storage and PV systems for the first time, which allows us to explore how they interact with one another.

Through the interface with Matpower we have access to the network configuration, buses, branches and generation points. The Matpower tool is on the utility's side in the co-simulation framework. Having the $24 \mathrm{~h}$ split into many control periods with Matpower being invoked at each of these, any changes or variations (due to say breaks in branches, or load changes due to the use of ESS) can be detected and used to generate newly updated demand response DR signals sent from the utility's site to targeted building owners. In this case, such signals represent signals meant to achieve load balancing in the network.

Our discussion here is under the assumption that the utility (i.e., Matpower tool, which deals with the distribution level calculations) continuously monitors the network and when faults that result in line breaks occur, the utility first runs a standard service restoration routine and then it addresses potential imbalances using the proposed balancing technique, as shown in Fig. 2 


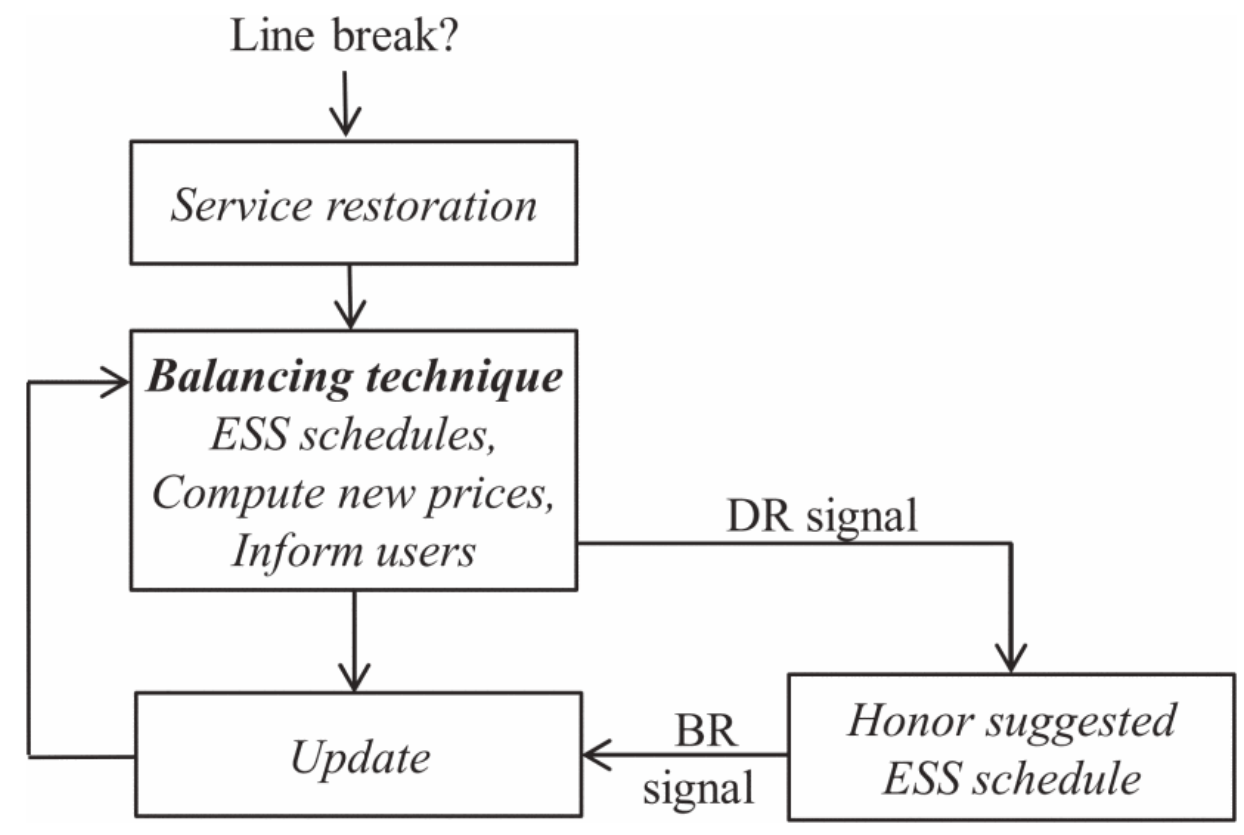

Fig. 2. Illustration of when the balancing technique is used.

The output of the balancing algorithm is a set of DR signals to request buildings within the network to reduce their power consumption by the supplied rate. Each building has the ability to use energy storage systems (ESS), PV systems, or smart building energy saving techniques to reduce its load. In this paper, we focus only on the use of ESS. Similarly to [19], we use an objective function given by the summation of the ratio of branch complex power to max allowed complex power throughout the network. This is used to determine system changes where the balancing technique is needed.

$$
\text { Objective }_{\text {cost }}=\sum \frac{P_{i}^{2}+Q_{i}^{2}}{\left(S_{i}^{\text {max }}\right)^{2}}
$$

The pseudocode description of the balancing algorithm is shown in Fig. 3. The main idea is to identify the network changes that occurred between two different iterations/runs of Matpower that cause imbalances that violate equation $\underline{1}$. When imbalances are detected, desired percentage load reductions are calculated for all buildings equipped with BESS. This is done using a simulated annealing (SA) algorithm ${ }^{[14]}$. The SA algorithm explores different load reduction rates on all BESS in the distribution network and identifies the best combination that minimizes the objective function from equation $\underline{1}$. The load reduction rates are sent as DR signals to all buildings. 


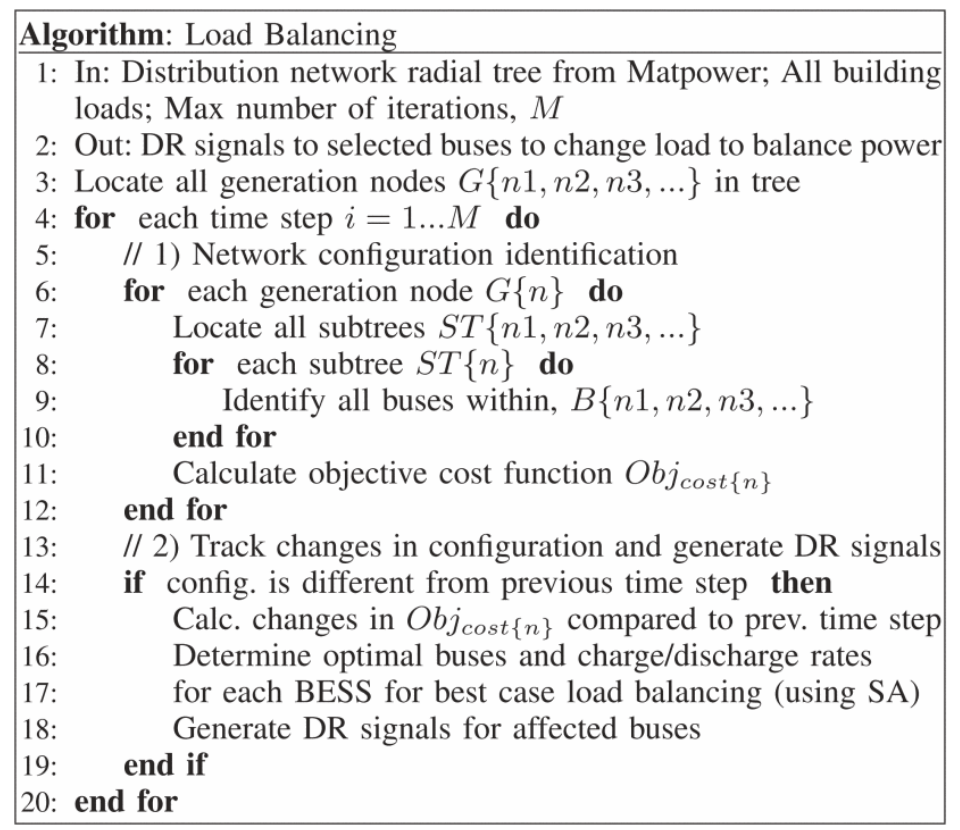

Fig. 3. Pseudocode of the load balancing algorithm used to determine load reduction rates requested from grid to offset temporary system changes.

\section{SECTION IV.}

\section{Simulation Results}

\section{A. Case Study: 9-Bus 16-Buildings Network}

Here, we report simulation results for a synthetic test case, which we created using a 9 bus test case whose topology is shown in Fig. 4. We modified this topology, which was included with Matpower, by removing all existing loads and adding loads at eight out of nine buses with custom loads consisting of clusters of two buildings per bus. Thus, the total number of buildings is 16 . Also, the generator data was sized to complement the loads on the system. In addition, each such cluster includes a BESS, which can be used by either of the two buildings attached to the same bus. The network is assumed to be located in Chicago in order to be able to use the EnergyPlus' standard weather file and modified reference buildings that are Chicago based.

We assume that this network topology has a tie switch as shown in the figure with dashed lines. In our testing setup, we assume that a fault occurs that breaks the indicated branch. As a result of having closed the tie switch to restore service to bus 7, an imbalance is introduced. In a larger distribution network, such disturbances can potentially have a larger impact, on outages or generational losses. The co-simulation framework has the ability to address such situations by interacting with smart buildings that have access to energy storage and PV systems in order to help balance the load. This interaction is accomplished through DR signals as discussed earlier.

The length of each simulation is set for $24 \mathrm{~h}$ with a time step of 5 minutes. Hence, the distribution network is simulated in Matpower at each step of 5 minutes, or 12 times per hour for a total of 288 
iterations or time steps. In this testing, the simulated line break fault is injected at time step 120. First, we present the simulation plots after the assumed line break and the service restoration is completed. In this case, there is no load balancing done. The plot of the objective function is shown in Fig. 5.a. This is for the subtree of the network that is more adversely affected. We can see that in the case when nothing is done for balancing, the objective cost function has a high peak for a long period.

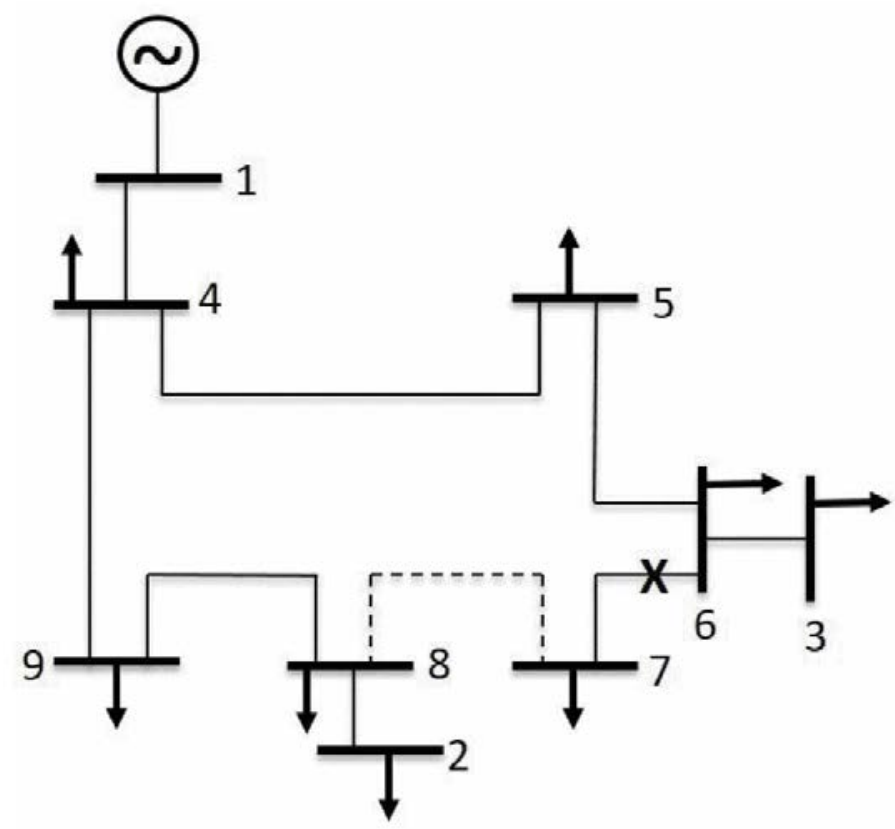

Fig. 4. Network topology of the 9 bus test case.

Then, we present the same simulation but with the balancing technique being applied. In this case, the plot of the objective function is shown in Fig. 5.b. We can see that the objective cost function was improved. This is due to the load adjustments at different buses based on the DR signals generated with the algorithm from Fig. 3. The DR signals are sent only to selected buildings that are requested to use their ESS for indicated time intervals. In this test case, the optimized BESS load reduction for buses 2-9 was determined to be respectively $[0.4,0.9,0.9,0.6,0.7,1.0,0.3,0.7]$. Please note that this improvement is achieved under the assumption that buildings would comply with the suggestions from the utility via the DR signals. In order to have such compliance (that cannot be guaranteed), the utility will have to resort to an incentive scheme and to possibly model and exploit user behavior to motivate building owners to cooperate with the utility.

\section{B. Case Study: 57-Bus 63-Buildings Network}

Here, we report simulation results for another synthetic test case, which we modified from a 63 building, 57 bus test case included with Matpower, whose topology is shown in Fig. 6 . Again, it is set in Chicago using EnergyPlus' included weather file and accompanying 16 Chicago based reference buildings. The simulation is also run for a $24 \mathrm{~h}$ period, with 12 steps per hour and a power flow being 
run at each step. Each load arrow represents a single building and BESS combo that will have the ability to communicate to the distribution network via DR signal.

This test case is set up similarly to the previous case. The length of each simulation is also set for $24 \mathrm{~h}$ with a time step of 5 minutes. The network has a tie switch indicated with a dashed line in Fig. 6 , and a fault that breaks a line is assumed to occur as indicated in the figure. The simulated line break fault is introduced at time step 120. The break islands or isolates 16 buses. Thus, when the tie switch is closed a large imbalance is introduced in the system as shown in the plot from Fig. 7.a. Then, in the second run of this simulation, the imbalance is addressed by using the BESS based balancing technique. In this case, the plot of the objective function is shown in Fig. 7.b, where we can see again that the objective cost function was greatly improved.

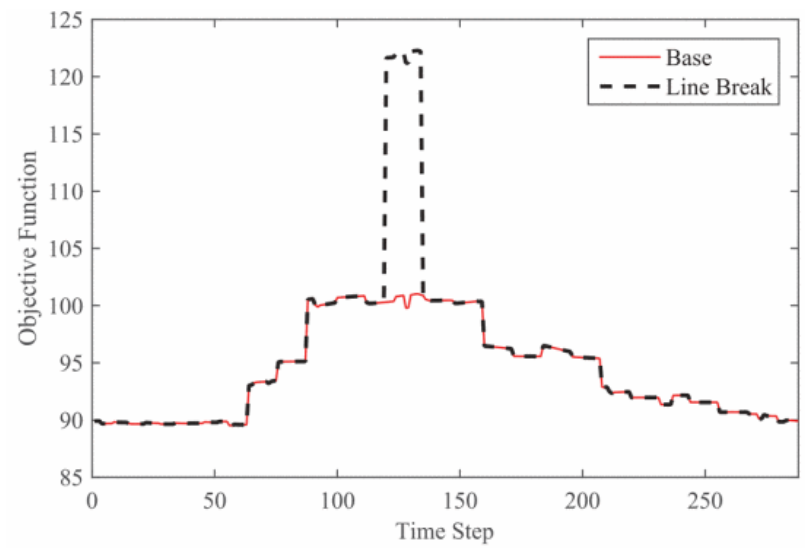

(a)

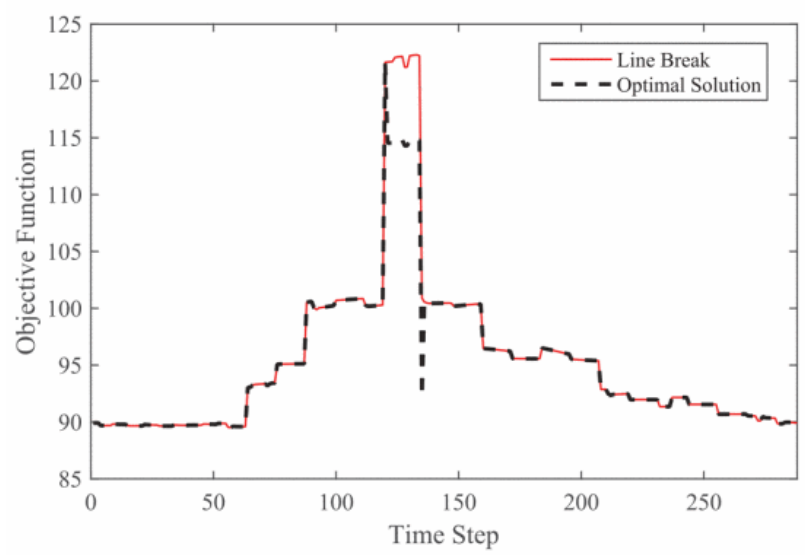

(b)

Fig. 5. A) objective cost function for the reference or base and imbalanced cases. B) imbalanced case versus balanced case.

The optimized BESS load reduction rate for all buses in the network, as calculated by the balancing algorithm, can be seen in Fig. 8. The figure is the solution space calculated from Fig. 3, where each bus and its corresponding load reduction rate is shown. Each of these load reduction rates are sent from the utility as DR signals to each building associated with the corresponding bus. Each building reduces 
their load impact, using BESS, by the decimal percentage received as a DR signal to achieve the network load balance shown in Fig. 7.b. The algorithm can successfully handle the 57-bus network in order to optimize the load reduction requirements for the objective cost function defined in equation $\underline{1}$.

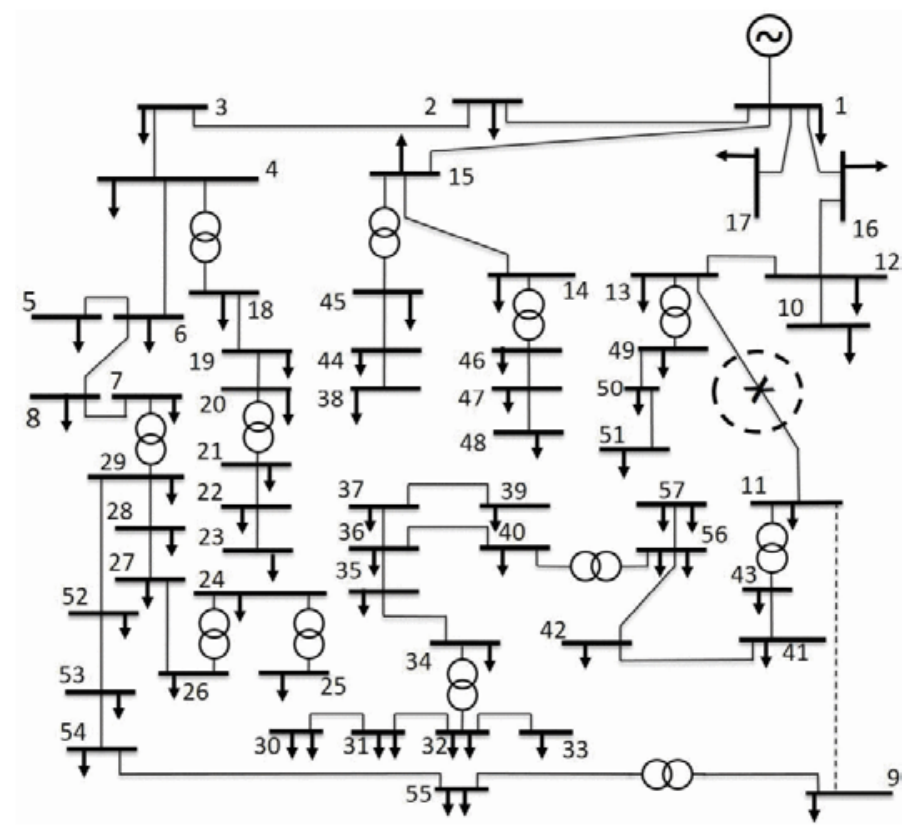

Fig. 6. Network topology of the 57 bus test case.

\section{SECTION V.}

\section{Conclusion}

We introduced a co-simulation framework that combines two main simulation tools, one that provides detailed multiple buildings energy simulation ability with EnergyPlus being the core engine, and the other one that is a distribution level simulator, Matpower. Exploiting the interaction between the distribution network (that is, the utility side) and multiple buildings (that is, the consumers side), it was shown that the proposed framework can offer a useful simulation platform for investigating district level optimization techniques. As an example, we investigated load balancing at the distribution network level in two separate test cases. 


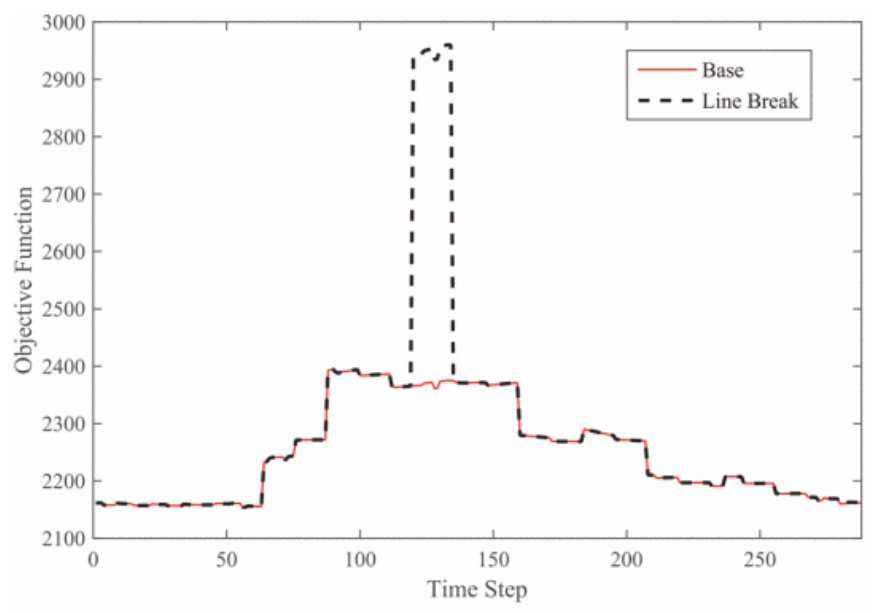

(a)

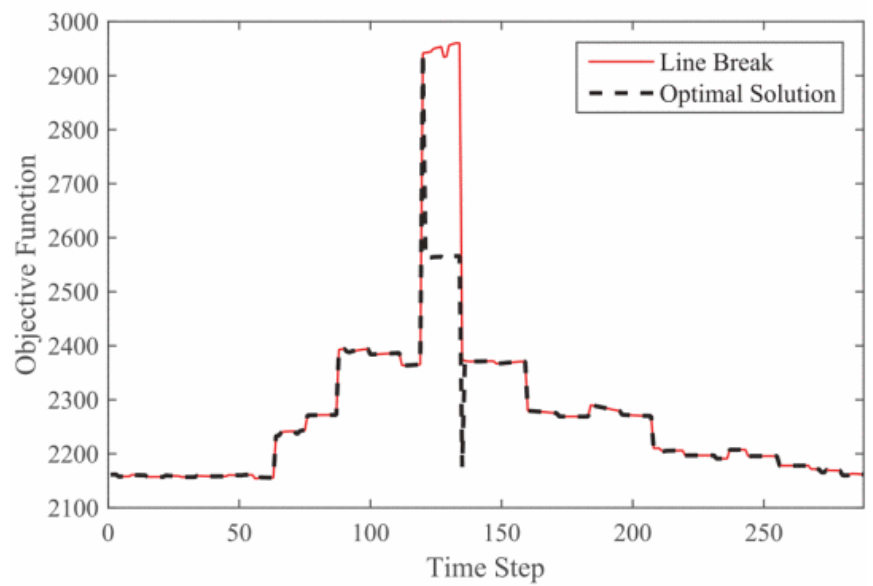

(b)

Fig. 7. A) objective cost function for the base and imbalanced cases. B) imbalanced case versus balanced case. 


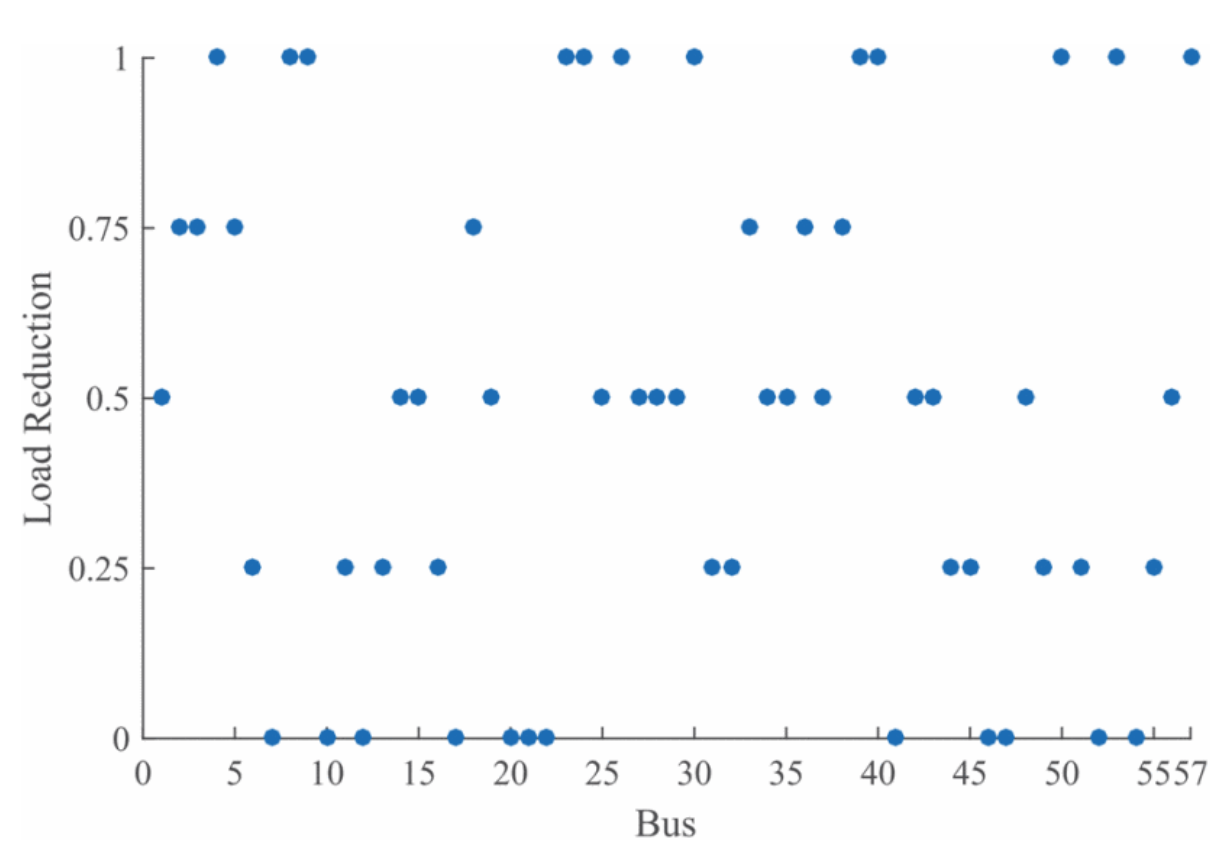

Fig. 8. Optimized BESS load reduction rates for 57-bus network.

\section{References}

1 Energy Star Facts and Stats, 2016, [online] Available: https://www.energystar.gov/buildings/aboutus/facts-and-stats.

2 United States Building Energy Efficiency Retrofits Report, 2012.

3 GRIDEDGE-utility modernization in the age of distributed generation, Oct. 2013, [online] Available: http://www.greentechmedia.com/gridedge.

4 C. F. Calvillo et al., "Distributed energy generation in smart cities", Int. Conference in Renewable Energy Research and Applications (ICRERA), pp. 161-166, Oct. 2013.

$5 \mathrm{~A}$. Tani et al., "Energy management in the decentralized generation systems based on renewable energy sources", Int. Conference in Renewable Energy Research and Applications (ICRERA), pp. 1-6, Oct. 2012.

6 A. K. Barnes, J. C. Balda, "Placement of distributed energy storage via multidimensional scaling and clustering", Int. Conference in Renewable Energy Research and Applications (ICRERA), pp. 69-74, Oct. 2014.

$7 \mathrm{M}$. H. Balali et al., "Development of an economical model for a hybrid system of grid PV and energy storage systems", Int. Conference in Renewable Energy Research and Applications (ICRERA), pp. 1108-1113, Oct. 2015.

8 B. Abedalsalam et al., "Microgrid communications: state of the art and future trends", Int. Conference in Renewable Energy Research and Applications (ICRERA), pp. 780-785, Oct. 2014.

9 EnergyPlus engineering reference, Sep. 2014, [online] Available:

http://appsl.eere.energy.gov/buildings/energyplus/pdfs/engineeringreference.pdf. 
10 R. D. Zimmerman et al., "MATPOWER: steady-state operations planning and analysis tools for power systems research and education", IEEE Trans. on Power Systems, vol. 26, no. 1, pp. 12-19, Feb. 2011.

$11 \mathrm{~J}$. Van Roy et al., "Ideas for tomorrow: new tools for integrated building and district modeling", IEEE Power and Energy Magazine, vol. 11, no. 5, pp. 75-81, Sep. 2013.

12 B. Aksanli et al., "Distributed control of a swarm of buildings connected to a smart grid", ACM Int. Conf. on Embedded Systems For Energy-Efficient Buildings (BuildSys), 2014.

13 B. Jiang, Y. Fei, "Smart home in smart microgrid: a cost-effective energy ecosystem with intelligent hierarchical agents", IEEE Trans. on Smart Grid, vol. 6, no. 1, pp. 3-13, Sep. 2014.

14 S. Duerr et al., "SmartBuilds: An energy and power simulation framework for buildings and districts", IEEE Trans. on Industry Applications, vol. 53, no. 1, pp. 402-410, 2017.

15 L. A. Hurtado et al., "Smart grid and smart building inter-operation using agent-based particle swarm optimization", Sustainable Energy Grids and Networks, vol. 2, pp. 42-50, 2015.

16 W. Bernal et al., "Campus-wide integrated building energy simulation", IBPSA Building Simulation Conf. Int. Building Performance Simulation, 2015.

17 T. X. Nghiem, "Design of the openBuildNet simulation (OBNSim) framework", Online Report, 2016.

$18 \mathrm{~W}$. Bernal et al., "MLE+: a tool for integrated design and deployment of energy efficient building controls", ACM Workshop on Embedded Sensing Systems for Energy-Efficiency in Buildings (BuildSys), 2012.

19 M. Baran, F. Wu, "Network reconfiguration in distribution systems for loss reduction and load balancing", IEEE Trans. on Power Delivery, vol. 4, no. 2, pp. 1401-1407, 1989. 